\title{
Photophysical and Photochemical Properties of Optical Oxygen Pressure Sensor of Platinum Porphyrin-Isobutylmethacrylate- Trifluoroethylmethacrylate Copolymer Film
}

\author{
Yutaka Amao, ${ }^{\dagger}$ Keisuke AsAI, Tokuji MiYashita, ${ }^{*}$ and Ichiro OKURA** \\ Aerodynamic Division, National Aerospace Laboratory, \\ Jindaiji-higashi, Chofu, Tokyo 182-8522, Japan \\ * Institute for Chemical Reaction Science, Tohoku University, \\ Katahira, Aoba-ku, Sendai 980-8577, Japan \\ ** Department of Bioengineering, Tokyo Institute of Technology, \\ Nagatsuta, Midori-ku, Yokohama 226-8501, Japan
}

(Received February 23, 1999)

KEY WORDS Optical Oxygen Pressure Sensor Platinum Octaethylporphyrin / Phosphorescence /
Oxygen sensing is finding application in chemical, clinical analysis and environmental monitoring, ${ }^{1-3}$ and is classified titration, ${ }^{4}$ amperometry, ${ }^{5}$ chemiluminescence, ${ }^{6}$ and thermoluminescence. ${ }^{7}$ The most popular is amperometry using an oxygen electrode ${ }^{5}$ in which the rate of diffusion of oxygen to the cathode is measured. This system has limited because of the stability of the electrode surface. Recently, a variety of devices and sensors based on photo-luminescence quenching of organic dyes, such as polycyclic aromatic hydrocarbons (pyrene and its derivatives, quinoline, and phenanthrene), ${ }^{8-10}$ transition metal complexes (ruthenium, ${ }^{11-13}$ osmium, ${ }^{14}$ or rhenium-polypyridine complexes $^{15}$ ), and metalloporphyrins, ${ }^{16-18}$ immobilized in oxygen permeable polymer (silicon polymer, polystyrene, and so on) has been developed to measure oxygen pressure on the solid surface. Platinum and palladium porphyrins show no more than weak prompt fluorescence $\left(\Phi_{\mathrm{F}}<10^{-3}\right)$ and phosphorescence yields $\Phi_{\mathrm{P}}$ variable over the entire range from $10^{-4}$ to 1 at room temperature. ${ }^{19-24}$ Phosphorescence lifetime $\tau_{\mathrm{P}}$ is short, typically less than $3.0 \mathrm{~ms}^{19}$ Especially, platinum octaethylporphyrin (PtOEP) displays strong room-temperature phosphorescence with high quantum yield $\left(\Phi_{\mathrm{P}}=0.5\right)$ and long lifetime $(c a .100 \mu \mathrm{s}) .{ }^{19}$ Some optical oxygen sensors based on phosphorescence quenching of PtOEP-polymer (polystyrene, silicone rubber, and so on) by oxygen have been developed. ${ }^{25,26}$ Organic dyes interact with polymer molecules and thus optical sensing properties strongly depend on the properties of polymer matrices. Oxygen permeable polymers with lower diffusion barrier for oxygen and higher stability for photo-oxidation are desired. Fluoro-polymer film allows large permeability of oxygen. ${ }^{27} \mathrm{C}-\mathrm{F}$ bond length is short $(13.17 \mathrm{~nm})$; bonding energy is large $\left(116 \mathrm{kcal} \mathrm{mol}^{-1}\right)$, compared with $\mathrm{C}-\mathrm{H}\left(99.5 \mathrm{kcal} \mathrm{mol}^{-1}\right)$ and electronegativity of fluorine is large, and thus polymers containing perfluoro-groups are stable forward photo-oxidation. Oxygen affinity is induced by large electronegativity of fluorine and oxygen permeability of the polymers containing perfluoro-group

\footnotetext{
† To whom correspondence should be addressed.
}

will be large. ${ }^{28-32}$ Polymers containing perfluorogroup are suitable for the above requirements.

In this work, polymethacrylate containing perfluorogroup, poly(isobutylmethacrylate-co-trifluoroethylmethacrylate) as shown in Figure 1, was synthesized and applied to the matrix of optical oxygen sensing using PtOEP.

\section{EXPERIMENTAL}

\section{Materials}

Isobutylmethacrylate (IBM), trifluoroethylmethacrylate (TFEM) and polyisobutylmethacrylate (poly-IBM) were purchased from Wako Pure Chemicals Co., Ltd. and were distilled under reduced pressure to remove the inhibitor. Azobis(isobutyronitrile) (AIBN) was obtained from Tokyo Chemical Industry Co. Ltd. and was recrystallized from ethanol. PtOEP was purchased from Porphyrin Products. All other chemicals were obtained from Wako Pure Chemicals Co., Ltd.

\section{Synthesis of Poly(isobutylmethacrylate-co-trifluoroethyl- methacrylate) (Poly-IBM-co-TFEM)}

Poly-IBM-co-TFEM was synthesized as follows. IBM $(0.16 \mathrm{~mol})$, TFEM $(0.15 \mathrm{~mol})$, and AIBN $(25 \mathrm{mmol})$ were dissolved in $80 \mathrm{~mL}$ toluene. The reaction mixture was heated at $80^{\circ} \mathrm{C}$ for $5 \mathrm{~h}$ under nitrogen atmosphere. After the mixture was cooled to room temperature, the polymer was precipitated in methanol. The solid was collected by filtration, washed with methanol to remove unreacted monomer and finally dried in vacuum. The composition ratio of IBM and TFEM was determined using the molar absorption coefficients of IBM and TFEM at $270 \mathrm{~nm}$. Molecular weight was determined by gel permeation chromatography (TSK HLC-802A TOYO SODA). IBM $/$ TFEM $=1.2$; GPC: $M_{n}=33870, M_{w}=58270$, and $M_{w} / M_{n}=1.72$.

\section{Preparation of Oxygen Sensing Film}

PtOEP-polymer film was formed by casting a mixture of $30 \mathrm{wt} \%$ polymer (poly-IBM-co-TFEM or polyIBM) and PtOEP in toluene onto non-luminescent glass 


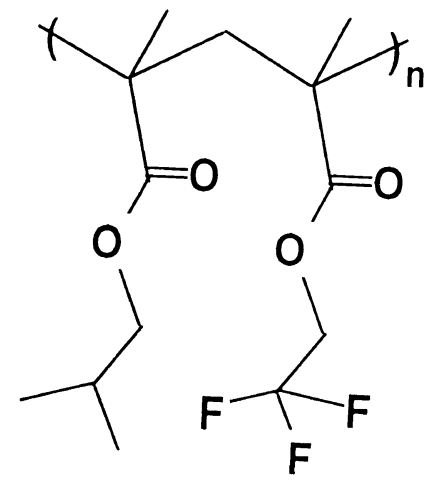

Figure 1. Chemical structure of polymethacrylate containing perfluoro-group, poly(isobutylmethacrylate-co-trifluoroethylmethacrylate) (poly-IBM-co-TFEM).

slides. PtOEP concentration in the film was approximately $2.9 \times 10^{-5} \mathrm{~mol} \mathrm{dm}^{-3}$. The films were dried at room temperature and stored in the dark prior to use. Film thickness was determined by a micron-sensitive caliper.

\section{Spectroscopic Measurement}

Absorption spectrum of PtOEP-polymer film was recorded using a Shimadzu U-2400PC spectrometer. Steady state phosphorescence spectra and excitation spectra were measured using a Shimadzu F-5300PC spectrofluorophotometer with a $150 \mathrm{~W}$ Xenon lamp as the visible excitation light source. Excitation and emission bandpasses were $5.0 \mathrm{~nm}$. All samples were excited at $535 \mathrm{~nm}$ attributed to the Q-band of PtOEP, the wavelength producing maximum phosphorescence intensity at $646 \mathrm{~nm}$.

\section{Oxygen Sensing System}

Oxygen sensing was carried out using a spectrofluorophotometer with a $150 \mathrm{~W}$ Xenon lamp as the visible excitation light source. Sample film was mounted at $45^{\circ}$ angle in the quartz cell to minimize light scatter from the sample and substrate. Different oxygen standards (in the range $0-100 \%$ ) in a gas stream were produced by controlling the flow rates of oxygen and argon gases entering the mixing chamber. Total pressure was maintained at 760 Torr. $^{25,26}$ PtOEP-poly-IBM film was used as a reference. All experiments were carried out at room temperature.

\section{RESULTS AND DISCUSSION}

\section{Photophysical Properties of PtOEP-Polymer Films}

Absorption spectra of PtOEP in poly-IBM and in poly-IBM-co-TFEM films were almost the same as in solution (absorption maxima $=534,501$, and $378 \mathrm{~nm}$ in the polymer; 535,500 , and $377 \mathrm{~nm}$ in toluene solution; data are not shown). This indicates no electrical interaction between PtOEP and polymer at the ground state.

Phosphorescence spectra of PtOEP-poly-IBM-coTFEM film under deoxygenated, ambient, and oxygenated conditions are shown in Figure 2, indicating quenching of the phosphorescence by oxygen. In the case of PtOEP-poly-IBM film as reference, quenching of the

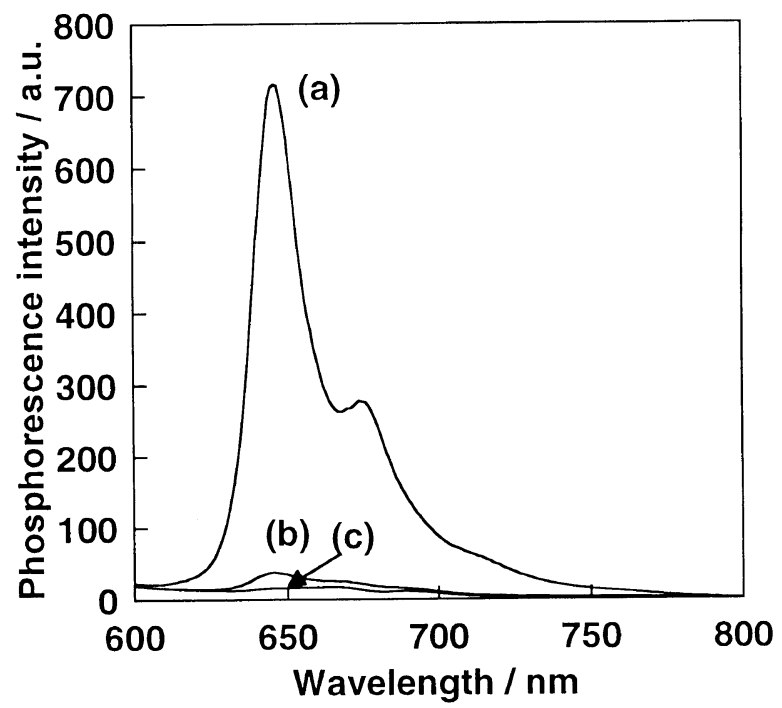

Figure 2. Phosphorescence spectrum change of PtOEP-poly-IBMco-TFEM film at various oxygen pressures. Excitation wavelength was $535 \mathrm{~nm}$. (a) deoxygenated, (b) ambient, and (c) oxygenated conditions.

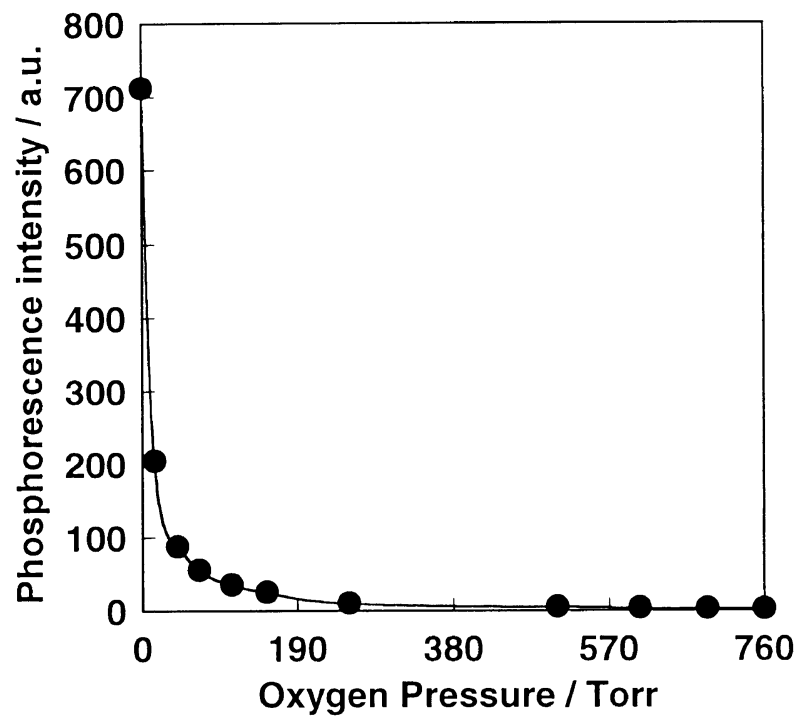

Figure 3. Relative phosphorescence intensity changes of PtOEP-poly-IBM-co-TFEM film at various oxygen pressures. Excitation and emission wavelength was 535 and $646 \mathrm{~nm}$, respectively.

phosphorescence by oxygen was observed. The phosphorescence spectra of PtOEP-poly-IBM-co-TFEM and PtOEP-poly-IBM films showed no red (or blue)-shift and no differences in peak shape compared to that of toluene solution $(646 \mathrm{~nm}$ in both instances). Phosphorescence maxima intensity increased strongly on going from oxygenated to ambient and deoxygenated conditions. The intensity of PtOEP-poly-IBM-co-TFEM film decreased with increase oxygen pressure as shown in Figure 3. This indicates that the phosphorescence intensity of PtOEP in poly-IBM-co-TFEM strongly depends on oxygen pressure and this film can be used for optical oxygen sensing based on phosphorescence quenching by oxygen. $I_{0} / I_{100}$ is used as sensitivity of sensing film, where $I_{0}$ and $I_{100}$ represent detected phosphorescence intensity from film exposed to $100 \%$ argon and $100 \%$ oxygen, respectively. A sensor with $I_{0} / I_{100}$ more than 3.0 is suitable for oxygen sensing. ${ }^{33}$ $I_{0} / I_{100}$ of poly-IBM-co-TFEM and poly-IBM film was 


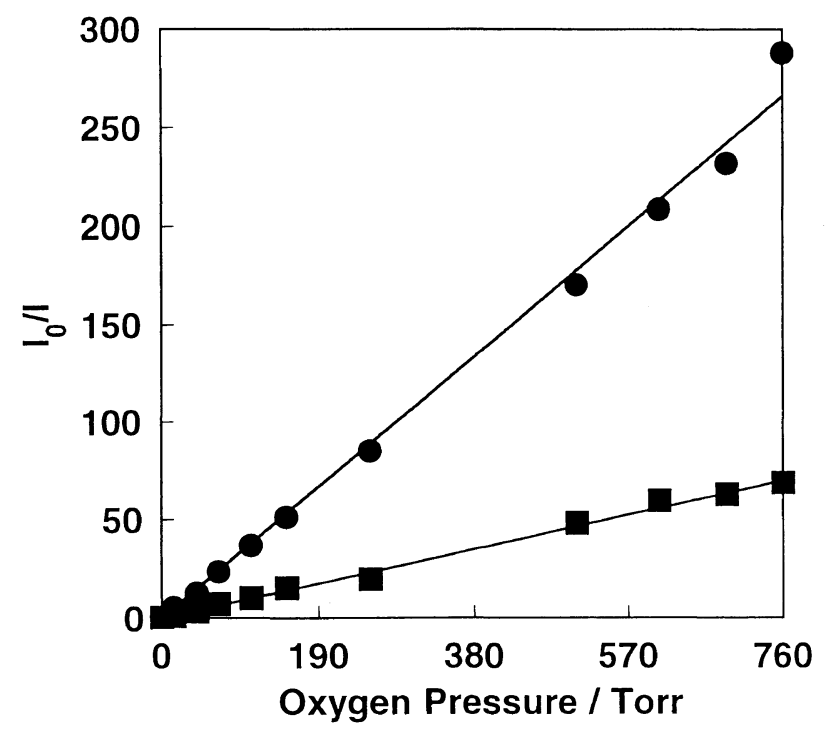

Figure 4. Stern-Volmer plot for PtOEP in poly-IBM-co-TFEM (-) and in poly-IBM (ם). Excitation and emission wavelengths were 535 and $646 \mathrm{~nm}$, respectively.

288 and 69.3, respectively. This indicates that PtOEPpoly-IBM-co-TFEM film is a highly sensitive device for oxygen.

\section{Oxygen Sensing Properties of PtOEP-Polymer Film}

Phosphorescence intensity from PtOEP in polymer film is quenched by oxygen according to the Stern-Volmer equation $\left(I_{0} / I=1+K_{\mathrm{Sv}} \mathrm{pO}_{2}\right.$; where $I_{0}$ and $I$ are phosphorescence intensity in the absence and presence of oxygen, respectively; $K_{\mathrm{Sv}}$ is the Stern-Volmer quenching constant. $\mathrm{pO}_{2}$ is oxygen pressure). Figure 4 shows Stern-Volmer plots of PtOEP-poly-IBM-co-TFEM and PtOEP-poly-IBM films. $K_{\mathrm{Sv}}$ of poly-IBM-coTFEM and poly-IBM films were obtained by least squares as 0.091 and $0.38 \mathrm{Torr}^{-1}$, respectively. In both polymers, the plots exhibit considerable linearity (correlation factor, $r^{2}=0.994$ for poly-IBM-co-TFEM film and 0.996 for poly-IBM film, respectively).

These polymers differ in the size and rigidity of side functional groups. In the case of poly-IBM-co-TFEM, interfacial intermolecular force between the gaseous phase and surface on polymer film is lower than that of poly-IBM, and oxygen affinity is induced by the large electronegativity of fluorine. ${ }^{28-32}$ Thus, large oxygen permeability of poly-IBM-co-TFEM is induced by lower surface free energy and larger electronegativity of fluorine. A highly sensitive optical sensor was developed using poly-IBM-co-TFEM as polymer matrix.

The effects of the composition ratio of IBM to TFEM and degree of polymerization on oxygen sensing are now being studied.

Acknowledgments. The present work is partly supported by a Grant-in-Aid for Priory-Areas-Research from the Ministry of Education, Science, Sports and Culture of Japan (No.10145211).

\section{REFERENCES}

1. C. Prininger, I. Klimant, and O.S. Wolfbeis, Anal. Chem., 66, 1841 (1994)

2. R. C. Martin, S. F. Malin, D. J. Bartnil, A. M. Schilling, and S. C. Furlong, Proc. SPIE., 2131, 426 (1994).

3. M. J. Atkinson, F. I. M. Thomas, N. Larson, E. Terrill, K. Morita, and C. C. Lium, Deep-Sea Res. I. 42, 761 (1995).

4. D. A. Skoog, D. M. West, and F.J. Holler, "Fundamentals of Analytical Chemistry," Saunders, Philadelphia, 1988, p 344.

5. L. C. Clark, Trans. Am. Artif. Intern. Organs., 2 , 41 (1956).

6. T. M. Freeman and W. R. Seitz, Anal. Chem., 53, 98 (1981).

7. H. D. Hendricks, US Patent, 3709663 (1973).

8. T. Ishiji and M. Kaneko, Analyst, 120, 1633 (1995).

9. A. Sharma and O. S. Wolfbeis, Appl. Spect., 42, 1009 (1988).

10. H. W. Kroneis and H. J. Marsoner, Sens. Actuators, 4, 587 (1983).

11. W. Xu, R. Schmidt, M. Whaley, J. N. Demas, B. A. DeGraff, E. K. Karikari, and B. L. Farmer, Anal. Chem., 67, 3172 (1995).

12. E. R. Carraway, J. N. Demas, B. A. DeGraff, and J. R. Bacon, Anal. Chem., 63, 332 (1991).

13. J. R. Bacon and J. N. Demas, Anal. Chem., 59, 2780 (1987).

14. W. Y. Xu, K. A. Kneas, J. N. Demas, and B. A. DeGraff, Anal. Chem., 68, 2605 (1996).

15. L. Sacksteder, J. N. Demas, and B. A. DeGraff, Anal. Chem., 65, 3480 (1993)

16. D. B. Papkovsky, G. V. Ponomarev, W. Trettnak, and P. O'Leary, Anal. Chem., 67, 4112 (1995).

17. J. Vanderkooi, G. Maniara, J. Green, and D. F. Wilson, J. Biol. Chem., 262, 5476 (1987).

18. A. Mills and A. Lepre, Anal. Chem., 69, 4653 (1997).

19. K. Kalyanasundaram, "Photochemistry of Polypyridine and Porphyrin Complexes," Academic Press, New York, N.Y., 1992, p 500 .

20. D. Eastwood and M. Gouterman, J. Mol. Spectrosc., 35, 359 (1970).

21. S. J. Lander and R. S. Becker, J. Chem. Phys., 43, 3344 (1965).

22. A. T. Gradyushko, V. A. Mashenkow, K. N. Solov'ev, and A.N. Sevchenko, Sov. Phys. Dokl., 13, 869 (1969).

23. J. B. Allison and R. S. Becker, J. Chem. Phys., 32, 1410 (1960).

24. R. S. Becker and J. B. Allison, J. Phys. Chem., 67, 262 (1963).

25. S-K. Lee and I. Okura, Anal. Sci., 13, 535 (1997).

26. S-K. Lee and I. Okura, Spectrochim. Acta A, 54, 91 (1998).

27. N. Yi-Yan, R. M. Felder, and W. J. Koros, J. Appl. Polym. Sci., 25, 1755 (1980).

28. W. A. Zisma, Advance in Chem. Series, 43, 1 (1964).

29. A. G. Pittman, "Fuluoropolymers," Wiley-Intersceince, New York, N.Y., 1972, p 446.

30. E. Wicke and E. U. Frank, Angew. Chem., 66, 701 (1954).

31. K. G. Denbigh, Trans. Faraday Soc., 36, 936 (1940).

32. A. Y. Kupryazhkin and E. V. Popof, Vysokomol. Soedin., Ser. B, 21, 287 (1976).

33. B. D. MacCraith, C. M. McDonagh, G. O'Keeffe, E. T. Keyes, J. G. Vos, B. O'Kelly, and J. F. McGilp, Analyst, 118, 385 (1993). 